\title{
In Situ Microscopic Observations of Magnetic Field Effects on the Growth of Silver Dendrites
}

\author{
Noriyuki Hirota ${ }^{1}$, Soma Hara $^{2}$ and Yoshio Sakka ${ }^{1}$ \\ ${ }^{1}$ Nano Ceramics Center, National Institute for Materials Science, Tsukuba 305-0047, Japan \\ ${ }^{2}$ Department of Advanced Materials Science, The University of Tokyo, Kashiwa 277-8561, Japan
}

\begin{abstract}
The effect of magnetic fields on electroless silver deposition was investigated through in situ microscopic observation using a periscope system developed on the basis of a confocal scanning laser microscope. At the growth front of a silver dendrite, under a $12 \mathrm{~T}$ magnetic field applied perpendicularly to the sample plane, a straight silver branch was grown for a while; then, a given length of the branch at the neighbor of the tip started moving rapidly and was bent in an integrated manner. As a result of the process, a dense silver dendrite in the shape of a vortex was formed. When the sample space was narrowed, the branch did not bend due to the increase in the static friction between the branch and the glass plates. Judging from these observations, the mechanism underlying the formation of a dense vortex dendrite was thought to be the effect of the Lorentz force acting on the branch due to the electric current flowing through the branch itself accompanied by the silver deposition and the copper dissolution reactions. Furthermore, the reason that the silver dendrite grown under high magnetic fields looked denser by macroscopic observation was investigated by evaluating the reaction amount and the microstructure of the silver branch. No significant difference was observed in the reaction amount between the experiments with and without magnetic fields. On the other hand, the microstructures of the silver branch were significantly different from each other. The silver branch grown under high magnetic fields had a low-density structure, which seems to account for its denser appearance in the macroscopic observation. This low-density structure may be formed because of the interference of the static crystal growth due to the rapid bend of the silver branches. [doi:10.2320/matertrans.MI200721]
\end{abstract}

(Received June 18, 2007; Accepted August 31, 2007; Published October 25, 2007)

Keywords: electroless silver deposition, confocal microscope, periscope, high magnetic fields, Lorentz force, in situ observation

\section{Introduction}

The recently achieved remarkable diffusion of a cryocooler-operated superconducting magnet is making it possible to easily use high magnetic fields in large-scale roomtemperature spaces. With these new developments, it has become popular to study the effects of magnetic fields on feeble magnetic materials or the processes involved in feeble magnetic materials. There have been many reports on the effects of magnetic fields on some crystal growth processes or materials processing. In some studies, high magnetic fields have been used in an attempt to control the morphology or structure of the materials. For example, it has been reported that the orientation of some ceramics, due to their magnetic anisotropy, was controlled by adopting a slip-casting method under high magnetic fields. ${ }^{1,2)}$ In the solidification process of some alloys or metals, inclusions can be isolated by utilizing the Lorentz force due to their difference in conductivity with the main matrix. ${ }^{3)}$ By utilizing magnetic dipole interactions in many-particle systems, some ordered alignments, such as chain-like alignments and triangle-lattice alignments, were obtained under high magnetic fields. ${ }^{4)}$

One of the remarkable magnetic field effects in the field of materials processing is the dramatic change in the morphology of the silver dendrite in the electroless silver deposition process. ${ }^{5)}$ When electroless silver deposition takes place in a very thin sample space, the shape of the silver dendrite is similar to that of a diffusion-limited aggregation (DLA) cluster. Branches of the dendrite are scattered and vary in length. Under high magnetic fields of several Teslas applied perpendicularly to the sample plane, the morphology of the silver dendrite changes into a dense vortex shape. The lengths of the branches in this case are almost uniform.

From a macroscopic observation, the formation of the dense morphology was attributed to the change in the ratedetermining process from a diffusion-limited process to a reaction-limited one, and the vortex shape was thought to be the result of the Lorentz force acting on the silver ions in the solution. The magnetic energy induced in feeble magnetic materials itself does not seem to be large enough to change the rate-determining process of a deposition reaction directly even under high magnetic fields. Another possibility for causing the change in the rate-determining process is the magneto-hydrodynamic (MHD) effect. If the flow is induced in the solution due to the MHD effect, it may change the materials flow and cause a change in the rate-determining process. However, even though the effect of the Lorentz force on the silver ion was considered, it seemed unlikely that a winding branch would grow as reported. According to the result of a Monte Carlo simulation that evaluated the morphological change in the DLA pattern under a rotating flow, ${ }^{6)}$ even if the flow of the solution were induced by the Lorentz force acting on the silver ions, the branch winding should be in the opposite direction to that observed.

Another question regarding this phenomenon is that the silver dendrite grown under high magnetic fields looks denser than that grown without magnetic fields by macroscopic observation. If the amount of deposited silver varies depending on the presence or absence of magnetic fields, the implication is that the deposition reaction rate is affected by the magnetic fields in some way.

The reported observations are interesting from the viewpoint of engineering. Further understanding of the mechanism of the magnetic field effect on the morphology of silver dendrites would provide important information that could be used to design how to control some processes, such as materials processing by magnetic fields, or to evaluate the effects of magnetic fields on chemical and biological 
reactions. We anticipate more details from microscopic observations of this phenomenon.

Therefore, in this study, in situ microscopic observations of electroless silver deposition were conducted under a $12 \mathrm{~T}$ magnetic field using a periscope system developed for use under high magnetic fields on the basis of the commercially available confocal scanning laser microscope. Furthermore, to investigate the reason that the silver dendrite grown under high magnetic fields appeared denser than that grown without magnetic fields, the reaction amount was evaluated using Inductively Coupled Plasma (ICP), and the microstructure of the silver branches was observed with a Scanning Electron Microscope (SEM).

\section{The Periscope System}

Understanding the mechanism of magnetic field effects is required for the adequate design of process control by magnetic fields. One potential way to investigate the mechanisms is the direct visualization of the phenomenon under high fields. However, special attention is required when making the visualization apparatus utilizable under fields as high as $10 \mathrm{~T}$ or more. For example, magnetic materials cannot be used as structural materials. The space in which high fields are generated is limited to the inside of a metal cylinder (magnet bore) with a diameter of about $100 \mathrm{~mm}$ at room temperature. Illumination is required for visualization, but fluorescent lights or lamps may not be used because they are affected by fields due to the Lorentz force.

One of the conventional methods for visualization in such environments is the utilization of a CCD image sensor. A camera consisting of a small CCD chip and optical lenses may be inserted into a field. Even though this method is effective and produces a sufficient amount of information, the resolution is limited to a maximum of several dozen micrometers. Observation at higher resolution may yield helpful information for further understanding of the mechanism of the effects of magnetic fields on some phenomena.

Higher resolution seems to be attained by the utilization of a scanning laser microscope system. On the basis of the considerations outlined above, we developed a periscope system to make in situ high-resolution observations under high magnetic fields. ${ }^{7}$ )

As a base system of the periscope, a commercially available confocal scanning laser microscope, Model VL2000 (Lasertec Corporation), was used. This product has three components, a microscope body, a scanning unit, and a control unit. The scanning unit includes a laser light source, a laser scanning mechanism, an optical deflector, a 1/4 lambda plate, a polarizing beam splitter, relay lenses, and a onedimensional CCD array. The scanning unit is attached to the microscope body through a trinocular tube that can easily be attached and removed. In the system developed here, the scanning unit was disconnected from the microscope body and set outside of the superconducting magnet because it contains many magnetic materials. The wavelength of the laser light was $410 \mathrm{~nm}$. The laser from the scanning unit was introduced into the inside of the magnet using the periscope. With this method, the control unit is usable. This system can be operated similarly to a commercial confocal scanning
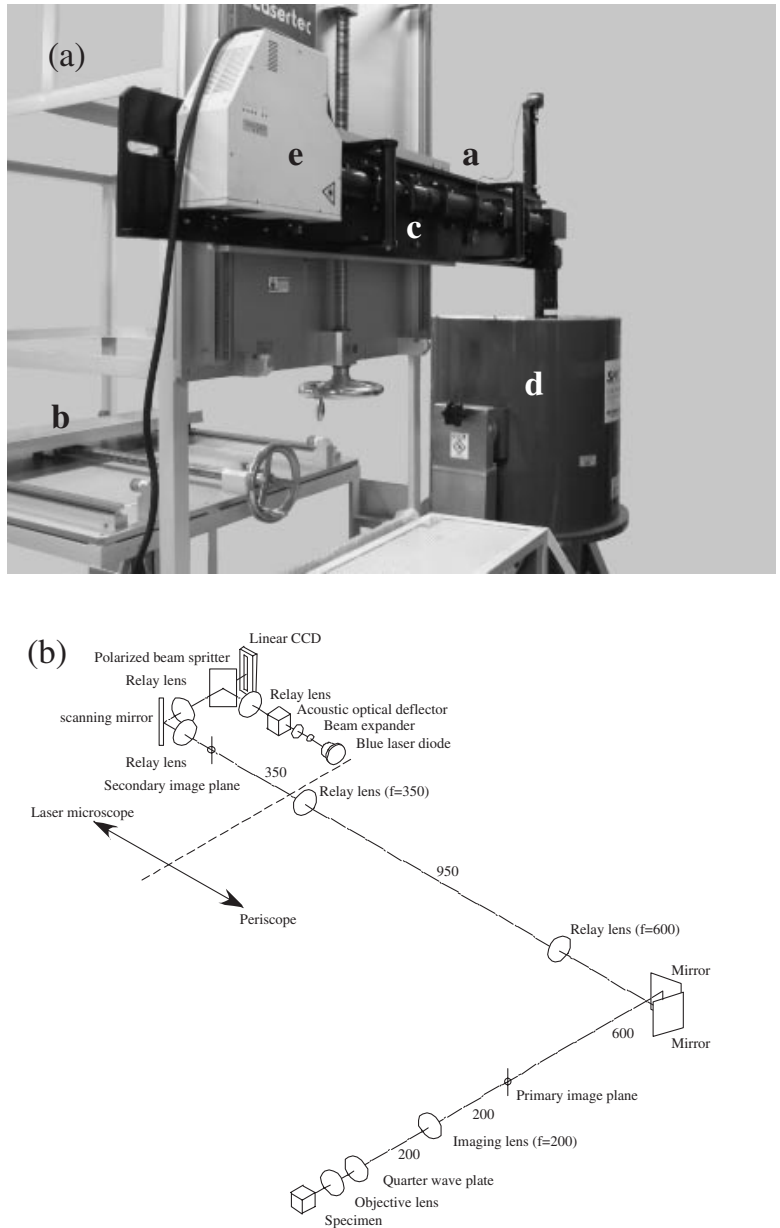

Fig. 1 Photograph of the periscope system and the superconducting magnet in a vertical setting (a) and scheme of the optical path of the periscope (b). a: stage to fix the periscope in a vertical position; $\mathbf{b}$ : stage to fix the periscope in a horizontal position; c: cylinder body of the periscope; d: superconducting magnet; e: scaning unit.

laser microscope. A photograph of the entire system is shown in Fig. 1(a). Figure 1(b) is a schematic illustration of the optical path of the periscope. The cylinder body of the periscope is made of Delrin.

The sample stage is fixed at the end of the periscope. On the sample holder, the flat sample and the optical cell of $10 \mathrm{~mm} \times 10 \mathrm{~mm} \times 30 \mathrm{~mm}$ are fixable. The sample stage is supported by the xyz fine-motion stage. Each stage is operated outside of the magnet using finger screws. The resolution obtained by this system was confirmed to be several hundreds nanometers. This resolution is the finest attained at this point by an optical observation system under high magnetic fields.

\section{Experimental}

The electroless silver deposition reaction is expressed in the following chemical equation:

$$
2 \mathrm{Ag}^{+}+\mathrm{Cu} \rightarrow 2 \mathrm{Ag} \downarrow+\mathrm{Cu}^{2+} .
$$

This reaction occurs when a piece of copper is introduced into a silver nitrate aqueous solution due to the difference in the oxidation-reduction potential. In the experiment here, this chemical reaction was carried out in a space sandwiched 
between two glass plates, and the behavior of the silver deposition was then observed in situ under a high magnetic field of $12 \mathrm{~T}$.

The size of the sample space was fixed to $18 \times 18 \mathrm{~mm}^{2}$, and the thickness was adjusted to $100 \mu \mathrm{m}$ or $3 \mu \mathrm{m}$ using a Teflon spacer between the two glass plates. The size of the copper metal sheets was $2 \times 2 \mathrm{~mm}^{2}$, and their thickness was $50 \mu \mathrm{m}$ or $2 \mu \mathrm{m}$ depending on the thickness of the sample space, $100 \mu \mathrm{m}$ or $3 \mu \mathrm{m}$, respectively. The concentration of the silver nitrate aqueous solution was 0.1 and $0.25 \mathrm{~mol} / \mathrm{dm}^{3}$ (this unit is equivalent with "mol/L").

First, a piece of copper was put in the sample space. After a few drops of the silver nitrate aqueous solution were placed into this space, the upper glass plate was set in place to form a closed system. The deposition reaction started immediately after the drop of the solution was placed. The sample was quickly introduced into the superconducting magnet. The magnet used in this study was a cryo-cooler operated type of superconducting magnet, model HF12-100VHT, manufactured by Sumitomo Heavy Industry Co., Ltd. This magnet has a room-temperature bore with a diameter of $100 \mathrm{~mm}$ and can produce $12 \mathrm{~T}$ magnetic fields at a maximum on the bore axis.

It took about 60 minutes to grow enough of the silver dendrite crystal under the experimental conditions used here. During this period, in situ observations of the silver dendrite growth were carried out under a magnetic field of $12 \mathrm{~T}$. To observe the entire shape of the dendrite, a CCD camera, model QN42 of ELMO Co., Ltd., was used. For the in situ microscopic observation of the growth front, a periscope system was used. Control experiments under a zero field were also carried out in the superconducting magnet without applying magnetic fields.

The amount of the electroless silver deposition reaction was determined by measuring the amount of dissolved copper in the solution after the silver dendrite growth. The amount of the deposited silver or decrease in the copper metal may be measured to evaluate the amount of the deposition reaction. However, the amount of the deposited silver seems difficult to measure. In evaluations by weight, it is difficult to collect all the deposited silver since some of the particles are very fine. When evaluating with ICP, it is very difficult to separate the deposited silver from the silver nitrate solution. Moreover, the amount of the copper metal decrement seems too small to evaluate by weighing. On the other hand, the measurement of the amount of dissolved copper ion into the solution seems to be the most precise way to evaluate the amount of the electroless silver deposition reaction.

The concentration of the silver nitrate aqueous solution used in this experiment was $0.25 \mathrm{~mol} / \mathrm{dm}^{3}$. The thickness of the sample space was $100 \mu \mathrm{m}$, and that of the copper metal sheets was $50 \mu \mathrm{m}$. Sixty minutes after the start of the reaction, the sample was quickly removed from the superconducting magnet. After the upper glass plate was carefully removed, the sample space, the upper glass plate, and the copper metal sheet were rinsed away with the solution using ultrapure water. The content of copper in this rinsed solution was measured using ICP (model Liberty AX Sequential ICPOES, Varian Co., Ltd.). The silver branch was collected after the dendrite growth, and its microstructure was observed with an SEM.
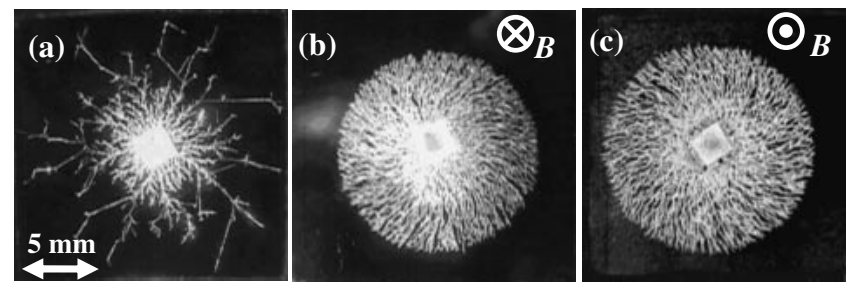

Fig. 2 Macroscopic observation of the morphology of a silver dendrite using a CCD camera. (a) under $0 \mathrm{~T}$, (b) $12 \mathrm{~T}$ applied perpendicularly to the space from the obverse side to the reverse side, (c) $12 \mathrm{~T}$ applied in the opposite direction to that in case (b).
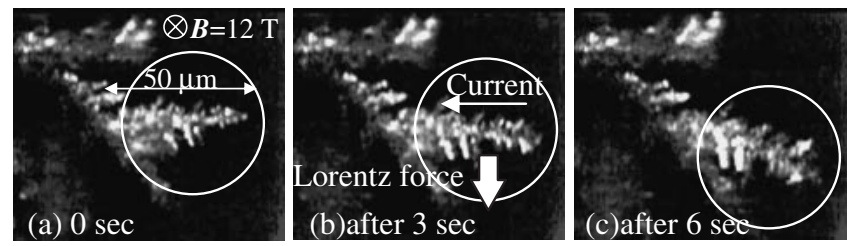

Fig. 3 In situ microscopic observation of the silver branch growth at the tip. These series of photographs were taken every 3 seconds from a given time. The magnetic field was applied $12 \mathrm{~T}$ perpendicularly to the space from the obverse side to the reverse side. The thickness of the sample space was set to $100 \mu \mathrm{m}$.

\section{Results and Discussion}

The results of macroscopic observation of the morphology of the silver dendrite obtained using a CCD camera are shown in Fig. 2. The thickness of the sample space was always $100 \mu \mathrm{m}$, and the concentration of the silver nitrate aqueous solution was $0.25 \mathrm{~mol} / \mathrm{dm}^{3}$. These photographs were taken 60 minutes after the start of the reaction. Without a magnetic field, the morphology of the silver dendrite became a typical DLA pattern, as shown in Fig. 2(a). Under a magnetic field of $12 \mathrm{~T}$ applied perpendicularly to the sample plane, it was confirmed, as reported, ${ }^{5)}$ that the morphology of the silver dendrite took a dense vortex shape and the direction of the branch winding was reversed depending on the direction of the magnetic field, as shown in Figs. 2(b) and (c).

Then, in situ microscopic observations of the growth front of the dendrite were carried out using the periscope system. In this experiment, a silver nitrate aqueous solution of $0.10 \mathrm{~mol} / \mathrm{dm}^{3}$ was used. The thickness of the sample space was $100 \mu \mathrm{m}$, and the applied magnetic field was $12 \mathrm{~T}$. Typical results of the observation are shown in Fig. 3 as a series of photographs of a growth front taken every 3 seconds from a given time. At the growth front, first, a straight silver branch was grown for a while; then, a given length of the branch at the neighbor of the tip started moving rapidly and was bent in an integrated manner. In the figure, the encircled branch was bent. This rapid bending of the silver branch was observed everywhere.

It is obvious that the Lorentz force plays an important role in this phenomenon because the direction of branch winding depends on the direction of the applied magnetic field. If, however, the vortex shape were formed due to the Lorentz force acting on the silver ion in the solution, a spirally winding branch should be formed from the beginning. 

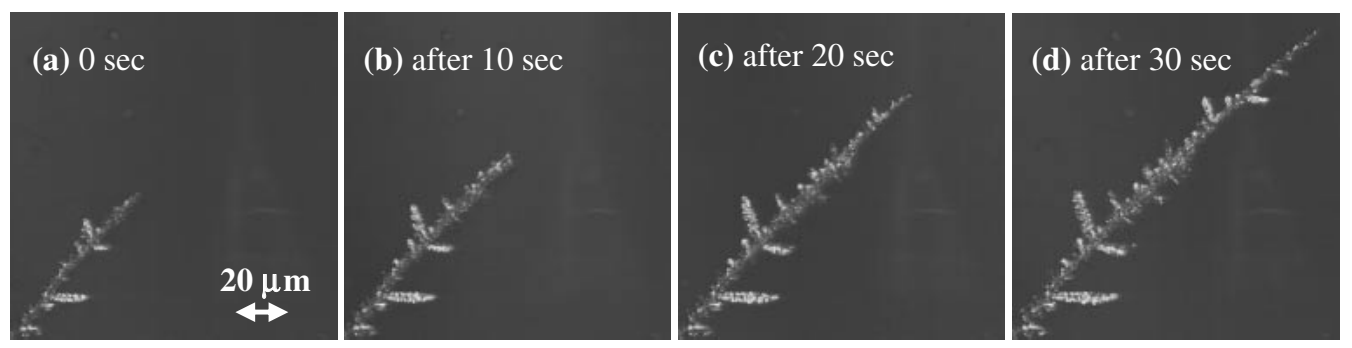

Fig. 4 In situ microscopic observation of the silver branch growth at the tip when the thickness of the sample space was set to $3 \mu \mathrm{m}$. These series of photographs were taken every 10 seconds from a given time. The magnetic field was applied $12 \mathrm{~T}$ perpendicularly to the space from the obverse side to the reverse side.

Judging from the observation here, this mechanism seems unlikely.

The points where silver deposition and copper dissolution occur are apart from each other and are connected electrically by silver branches. Therefore, the electric current flows in silver branches due to the transfer of electrons accompanied by the progress of the reaction. Because the direction of this current flow is perpendicular to the applied magnetic field, the Lorentz force acts on branches as a conductor. The direction of branch winding agrees with the direction of the Lorentz force. The mechanism of the rapid bending of branches is thought to be the effect of the Lorentz force acting on the branch due to the flow of the electric current.

The intensity of the Lorentz force acting on a silver branch observed in the case of Fig. 3 was estimated. ${ }^{8)}$ The intensity of the Lorentz force acting on the bent silver branch by the deposition of silver at a tip during one second was estimated to be $15 \%$ of the gravity force acting on the branch. Considering that the bent silver branch had several tips and silver deposition took place simultaneously at various sites, it is possible that a fairly large Lorentz force was acting on this branch.

The silver branch appeared to be at the bottom of the silver nitrate aqueous solution, touching the glass surface, because it was much heavier than the solution. When the Lorentz force acting on the branch was smaller than the static friction between the branch and the glass surface, the branch did not move. As the growth front increased, the Lorentz force became gradually larger because of the increase in the number of deposition sites. When the Lorentz force exceeded the static friction, the branch appeared to start moving and bend rapidly.

According to this hypothesis, the bending of silver branches should be suppressed due to the increase of the static friction when the space between the two glass plates containing the sample is narrow and the silver branches touch glass on both sides. From the result of the experiments, the approximate diameter of a silver branch seems to be several micrometers; therefore, the distance between the two glass plates was $3 \mu \mathrm{m}$ in the following experiment. The results obtained by the periscope system are shown in Fig. 4. In this figure, a series of photographs of a growth front taken every 10 seconds from a given time is shown as a typical result of the observation. The concentration of the silver nitrate aqueous solution was $0.25 \mathrm{~mol} / \mathrm{dm}^{3}$, and the applied magnetic field was $12 \mathrm{~T}$. Under this condition, silver branches grew straight, unlike those shown in Fig. 3. The macroscopic
Table 1 Amount of dissolved copper in the solution after the electroless silver deposition reaction measured using ICP.

\begin{tabular}{lc}
\hline & Amount of dissolved copper $(\mu \mathrm{g})$ \\
\hline $0 \mathrm{~T}$ & 245 \\
$12 \mathrm{~T}$ & 254 \\
\hline
\end{tabular}

observation of the morphology of the silver dendrite with the use of a CCD camera indicated that the branches were scattered; in addition, the formation of the vortex shape was not observed even under the magnetic field. No significant difference was observed from the results obtained under a zero field. Even though the Lorentz force was acting on the branches, their movement was assumed to be impeded by the anticipated increments of static friction. This observation also suggests the involvement of the bending of branches in the formation of a dense morphology. The bending of the branches suppresses the broadening of the dendrite, and the dendrite seems to be densely formed as a result.

Table 1 shows the measured amount of copper dissolved in the solution after the electroless silver deposition reaction for 60 minutes. Even under a magnetic field of $12 \mathrm{~T}$, no significant difference was observed in the amount of the reaction from the results obtained under $0 \mathrm{~T}$. In general, the energy generated by the magnetic fields is much smaller than the activation energy of chemical reactions. Therefore, it is unlikely that the magnetic fields accelerate chemical reactions. For this reason, the result obtained here seems appropriate. However, the question of why the silver dendrite grown under a magnetic field of $12 \mathrm{~T}$ looks denser in macroscopic observation, as shown in Fig. 2, must be asked. Photographs of the microstructure of the silver branches observed by SEM are shown in Fig. 5. Figure 5(a) shows the microstructure of the silver branch grown under $0 \mathrm{~T}$, and (b) shows that grown under $12 \mathrm{~T}$. The magnification ratios of these photographs are the same; however, there are significant differences in their microstructures. Without a magnetic field, static crystal growth occurred, and the silver branch consisted of some large crystals. On the other hand, under $12 \mathrm{~T}$ magnetic fields, the silver branch has porous, lowdensity structures. It was confirmed that the formation of such low density structures is the reason for the denser appearance in the macroscopic observation. One possible mechanism for the formation of such a low-density structure may be the interference of static crystal growth due to the rapid bend of the silver branches under high magnetic fields. 

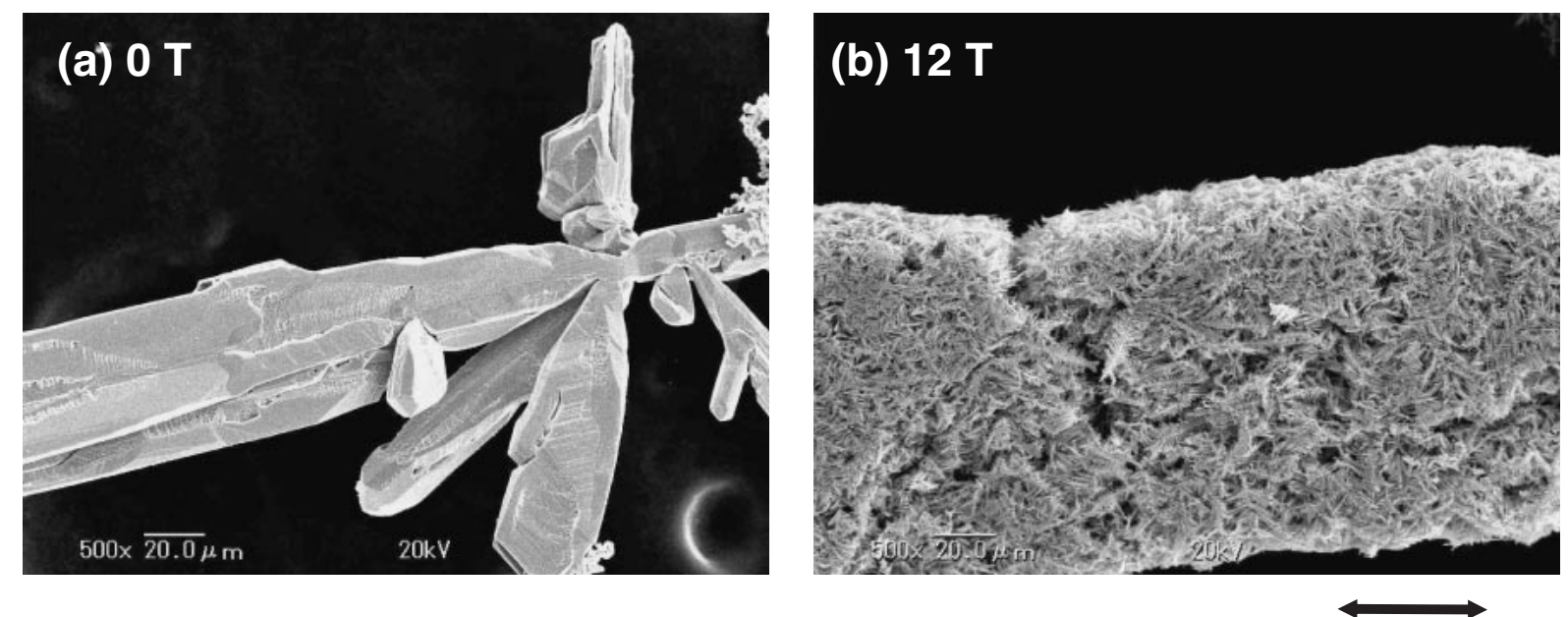

$50 \mu \mathrm{m}$

Fig. 5 Microstructure of the silver dendrite observed using a scanning electron microscope. (a) grown under $0 \mathrm{~T}$, (b) grown under $12 \mathrm{~T}$; concentration of the silver nitrate aqueous solution, $0.25 \mathrm{~mol} / \mathrm{dm}^{3}$; reaction time, 60 minutes.

For further understanding of this mechanism, we may need to consider the effect of the micro MHD flow in the solution reported by Katsuki et al. ${ }^{9)}$

\section{Conclusion}

In this study, to understand the mechanism of the magnetic field effect on the morphology of silver dendrite reported so far, an in situ microscopic observation of the electroless silver deposition process under a $12 \mathrm{~T}$ magnetic field was carried out using a periscope system. Observations under a $12 \mathrm{~T}$ magnetic field showed that the branch grew straight for a while on the growth front; then, a given length of the branch at the neighbor of the tip started moving rapidly and was bent in an integrated manner. As a result, the silver dendrite acquired a dense vortex shape. Then, in an experiment in which the sample space was narrowed to increase the static friction between the branches and the glass plates, straight silver branches grew. No significant difference was observed from the results obtained under a zero field, that is, a scattered dendrite was observed. Based on these observations, we concluded that the mechanism of the formation of a vortex morphology under a magnetic field was the effect of the Lorentz force acting on the silver branch due to the electric current flowing through the branch itself accompanied by the silver deposition and the copper dissolution reactions. It was also suggested that the bending of branches was involved in the formation of a dense morphology in the macroscopic observation through the suppression of the broadening of the dendrite. From the quantitative analysis of dissolved copper after the electroless silver deposition reaction, it was confirmed that there are no significant differences in the amount of the reaction even under high magnetic fields from the case without a magnetic field. It was also confirmed that the silver branch that was grown under $12 \mathrm{~T}$ magnetic fields has porous, low-density structures. The formation of such low-density structures is the reason for the denser appearance in the macroscopic observation. The observations in this study suggest that the morphology or structure of materials may be controlled in some materials processing that includes electrolysis or electro-crystallization as part of the process by the utilization of high magnetic fields. For example, use of magnetic fields may be effective for producing some catalytic substances in an electrochemical manner. The use of magnetic fields is characteristic for its non-contact manner, i.e., magnetic fields can have an effect on materials processing without having any direct contact with the matter. Therefore, the information obtained in this study seems useful to design a novel way to control materials processing using magnetic fields.

\section{REFERENCES}

1) T. S. Suzuki, Y. Sakka and K. Kitazawa: Advanced Engineering Materials 3 (2001) 490.

2) S. Horii, I. Matsubara, M. Sano, K. Fujie, M. Suzuki, R. Funahashi, M. Shikano, W. Shin, N. Murayama, J. Shimoyama and K. Kishio: Jpn. J. Appl. Phys. 42 (2003) 7018.

3) J. P. Park, K. Sassa and S. Asai: J. Jpn. Inst. Metals 59 (1995) 733.

4) T. Takayama, Y. Ikezoe, T. Kaihatsu, H. Uetake, N. Hirota and K. Kitazawa: Trans. Mater. Res. Soc. Jpn. 27 (2002) 43.

5) I. Mogi, S. Okubo and Y. Nakagawa: J. Phys. Soc. Jpn. 60 (1991) 3200.

6) T. Nagatani and F. Sagues: J. Phys. Soc. Jpn. 59 (1990) 3447.

7) N. Hirota and T. Ode: Rev. Sci. Instrum. 77 (2006) 036107.

8) N. Hirota, S. Hara, H. Uetake, H. Nakamura and K. Kitazawa: J. Crystal Growth 286 (2006) 465-469.

9) A. Katsuki and Y. Tanimoto: Chem. Lett. 34 (2005) 31. 